\title{
Adjunctive Brivaracetam in Focal Epilepsy: Real-World Evidence from the BRIVAracetam add-on First Italian netwoRk STudy (BRIVAFIRST)
}

\author{
Simona Lattanzi ${ }^{1}$ - Laura Canafoglia ${ }^{2}$. Maria Paola Canevini ${ }^{3,4} \cdot$ Sara Casciato ${ }^{5}$. Valentina Chiesa ${ }^{3}$. \\ Filippo Dainese $^{6}$. Giovanni De Maria ${ }^{7}$. Giuseppe Didato ${ }^{8}$. Giovanni Falcicchio ${ }^{9} \cdot$ Martina Fanella $^{10}$. \\ Edoardo Ferlazzo ${ }^{11}$. Giacomo Fisco ${ }^{10}$. Massimo Gangitano ${ }^{12}$. Anna Teresa Giallonardo ${ }^{10}$. Filippo Sean Giorgi ${ }^{13,14}$. \\ Angela La Neve ${ }^{9}$. Oriano Mecarelli ${ }^{10}$. Elisa Montalenti ${ }^{15} \cdot$ Federico Piazza $^{16}$. Patrizia Pulitano ${ }^{10}$. \\ Pier Paolo Quarato ${ }^{5}$. Federica Ranzato ${ }^{17}$. Eleonora Rosati ${ }^{18}$. Laura Tassi ${ }^{19}$. Carlo Di Bonaventura ${ }^{10}$ on behalf of \\ BRIVAFIRST Group Membership
}

Accepted: 9 August 2021 / Published online: 2 September 2021

(C) The Author(s) 2021, corrected publication 2021

\begin{abstract}
Background In randomized controlled trials, add-on brivaracetam (BRV) reduced seizure frequency in patients with drugresistant focal epilepsy. Studies performed in a naturalistic setting are a useful complement to characterize the drug profile. Objective This multicentre study assessed the effectiveness and tolerability of adjunctive BRV in a large population of patients with focal epilepsy in the context of real-world clinical practice.

Methods The BRIVAFIRST (BRIVAracetam add-on First Italian netwoRk STudy) was a retrospective, multicentre study including adult patients prescribed adjunctive BRV. Patients with focal epilepsy and 12-month follow-up were considered. Main outcomes included the rates of seizure-freedom, seizure response ( $\geq 50 \%$ reduction in baseline seizure frequency), and treatment discontinuation. The incidence of adverse events (AEs) was also considered. Analyses by levetiracetam (LEV) status and concomitant use of strong enzyme-inducing antiseizure medications (EiASMs) and sodium channel blockers (SCBs) were performed.

Results A total of 1029 patients with a median age of 45 years (33-56) was included. At 12 months, 169 (16.4\%) patients were seizure-free and $383(37.2 \%)$ were seizure responders. The rate of seizure freedom was $22.3 \%$ in LEV-naive patients, $7.1 \%$ in patients with prior LEV use and discontinuation due to insufficient efficacy, and $31.2 \%$ in patients with prior LEV use and discontinuation due to AEs $(p<0.001)$; the corresponding values for $\geq 50 \%$ seizure frequency reduction were $47.9 \%$, $29.7 \%$, and $42.8 \%(p<0.001)$. There were no statistically significant differences in seizure freedom and seizure response rates by use of strong EiASMs. The rates of seizure freedom $(20.0 \%$ vs. $16.6 \% ; p=0.341)$ and seizure response $(39.7 \%$ vs. $26.9 \% ; p=0.006$ ) were higher in patients receiving SCBs than those not receiving SCBs; 265 (25.8\%) patients discontinued BRV. AEs were reported by $30.1 \%$ of patients, and were less common in patients treated with BRV and concomitant SCBs than those not treated with SCBs $(28.9 \%$ vs. $39.8 \% ; p=0.017)$.

Conclusion The BRIVAFIRST provided real-world evidence on the effectiveness of BRV in patients with focal epilepsy irrespective of LEV history and concomitant ASMs, and suggested favourable therapeutic combinations.
\end{abstract}

\section{Introduction}

The members of the "BRIVAFIRST Group Membership" are listed in Acknowledgement section.

Simona Lattanzi

alfierelattanzisimona@gmail.com

Extended author information available on the last page of the article
Antiseizure medications (ASMs) play a central role in the treatment of people with epilepsy. Although several medications became available in the last decades, the prevalence of refractory epilepsy remains high and around one-third of patients continue to have incomplete seizure control [1, 2]. Due to ongoing seizures, patients often experience psychological dysfunction, social stigma, reduced educational 


\section{Key Points}

Adjunctive brivaracetam (BRV) improved seizure frequency in patients with focal epilepsy irrespective of levetiracetam history.

Seizure response was higher among patients treated with BRV and concomitant sodium channel blockers (SCBs).

Adverse events were less frequently observed when BRV was concomitantly administered with SCBs than with no SCBs.

and employment opportunities, impaired quality of life, and increased mortality [3]. Therefore, the development of new therapeutic options that are effective and safe is needed.

Brivaracetam (BRV) is a rationally developed compound characterized by high-affinity binding to synaptic vesicle protein 2A (SV2A) and chemical structure similar to levetiracetam (LEV) [4]. BRV is highly lipid-soluble, comparable with benzodiazepines and phenytoin. It is almost completely absorbed after oral administration and enters the brain rapidly by crossing the blood-brain barrier via passive diffusion $[5,6]$. BRV is weakly $(<20 \%)$ bound to plasma proteins and is rapidly and evenly distributed in most tissues. The pharmacokinetics of BRV are dose-proportional within the range of $10-600 \mathrm{mg}$. The drug is eliminated primarily by metabolism and by excretion in the urine, and the plasma half-life is approximately $9 \mathrm{~h}[5,6]$. BRV is primarily metabolized by hydrolysis of the acetamide group to the corresponding carboxylic acid metabolite, and secondarily by hydroxylation on the propyl side chain to form the hydroxy metabolite [5, 6]. The hydrolysis is mediated by hepatic and extrahepatic amidase, and the hydroxylation is mediated primarily by cytochrome P450 (CYP) 2C19. An additional hydroxy acid metabolite is produced by hydrolysis of the amide moiety on the hydroxy metabolite or hydroxylation of the propyl side chain on the carboxylic acid metabolite mainly through CYP2C9. None of the three main metabolites, namely acid, hydroxy, and hydroxy acid, are pharmacologically active $[5$, 6].

BRV does not interact with most drug-metabolizing enzymes and drug transporters, and is associated with few clinically relevant drug-drug interactions (DDIs) [7]. The effect of BRV on other ASMs has been evaluated in a meta-analysis of five pivotal trials [8]. No clinically relevant changes in plasma concentrations have been observed and no dose adjustment was needed when BRV at doses of up to $200 \mathrm{mg} /$ day was added to common ASMs (carbamazepine, lacosamide, lamotrigine, LEV, oxcarbazepine, phenobarbital, phenytoin, pregabalin, topiramate, valproate, and zonisamide) [7]. One exception was the dose-dependent increased exposure to carbamazepine-epoxide, the active metabolite of carbamazepine, due to BRV-mediated inhibition of epoxide hydrolase, and the effect size was not influenced by concomitant valproic acid [7]. In a post hoc analysis of clinical trials of adjunctive BRV, this inhibition has not been found to have clinically significant effects on safety or efficacy [9]; dose reduction of carbamazepine should however be considered if tolerability issues arise in patients receiving concomitant BRV. In a population pharmacokinetic modelling study of adults with focal seizures who participated in pivotal trials, carbamazepine, phenytoin and phenobarbital/primidone, which have strong enzymeinduction properties on many enzymes, decreased BRV levels by $26 \%, 21 \%$ and $19 \%$, respectively, with no significant influence on clinical response [10]. Dose adjustment and an increase in BRV dose of up to $100 \%$ should be considered when it is coadministered with the potent CYP2C19 inducer rifampin [7]. St. John's wort (Hypericum perforatum) is also a strong CYP inducer that may decrease BRV levels, and caution is needed when this herbal supplement is added or ended [7]. As BRV did not significantly inhibit or induce CYP3A and had no effects on midazolam metabolism, it would not be expected to interact with the oral anticoagulants apixaban and rivaroxaban [7]. BRV has been shown to modestly inhibit CYP2C19 in studies in human liver microsomes, and the potential increase in plasma levels of drugs primarily metabolized by CYP2C19 (e.g., diazepam, lansoprazole, omeprazole) cannot be excluded [7]. Furthermore, BRV weakly inhibits phenytoin metabolism through CYP2C19 inhibition in in vitro studies, and a pharmacokinetic study in patients with epilepsy demonstrated that BRV administered at the supratherapeutic dose of $400 \mathrm{mg} /$ day increased phenytoin exposure by approximately $20 \%$ [11]. There is evidence suggesting that BRV can be safely coadministered with inhibitors of CYP2C 8 or CYP2C9, and BRV exposure should not be significantly affected by coadministration with any CYP2C19 inhibitor [7, 12]. Furthermore, in vitro studies demonstrated no significant inhibition of drug transporters [7]. The overall low propensity of DDIs, which may be partly attributed to the presence of multiple elimination pathways and the major elimination route occurring through amidases, for which DDIs have not been reported, may allow the administration of BRV without dose adjustment and avoid potential untoward events [7].

In randomized, placebo-controlled trials, BRV reduced seizure frequency when added to pre-existing ASMs in patients with drug-resistant focal epilepsy [13]. The aim of this study was to assess the effectiveness and tolerability of adjunctive BRV over a 1-year period in a large population of patients with focal epilepsy treated in the context of realworld clinical practice. 


\section{Methods}

\subsection{Participants}

The BRIVAFIRST (BRIVAracetam add-on First Italian netwoRk STudy) was a retrospective study conducted across 62 Italian centres. Adult (age $\geq 16$ years) patients attending participating centres who were prescribed BRV (March 2018-March 2020) and were receiving stable treatment with one or more ASMs during the prior 90 days were retrospectively identified. Only patients with focal epilepsy and with 12-month follow-up after initiating BRV were included in the current analysis. Data on demographics, clinical history, type of seizures and epilepsy [14], etiology, previous/concomitant ASMs, and baseline seizure frequency (monthly seizure frequency during the 3 months before starting BRV) were collected. Concomitant ASMs were classified, according to their mechanism of action, into sodium channel blockers (SCBs; carbamazepine, phenytoin, lamotrigine, oxcarbazepine, eslicarbazepine acetate, lacosamide, rufinamide) and no SCBs (any other ASM); patients in the SCB group were those receiving at least one $\mathrm{SCB}$, whereas those in the no SCB group did not take any SCB. Concomitant ASMs were classified as strong enzyme-inducing ASMs (EiASMs), i.e., carbamazepine, phenytoin, phenobarbital, and primidone, being the major EiASMs that stimulate the rate of metabolism of most coadministered ASMs [15], and no strong EiASMs (any other ASM); patients were described as taking strong EiASMs if they were taking at least one strong EiASM during BRV treatment. Prospectively collected data on seizure occurrence, adverse events (AEs) and drug withdrawal were retrieved from patient seizures diaries and clinical records; visits at 3, 6, and 12 months were performed as standard practice when a new ASM was initiated. Exclusion criteria were history of alcoholism, drug abuse, conversion disorders or other non-epileptic ictal events.

Effectiveness outcomes included the rates of seizure freedom, seizure response ( $\geq 50 \%$ reduction in baseline monthly seizure frequency), seizure worsening ( $>25 \%$ increase in monthly seizure frequency relative to baseline, unless patients were seizure-free during the 3 months before starting BRV) and treatment discontinuation at 12 months. Further analyses were performed using data obtained from the visits at 3 and 6 months. Seizure freedom at each time point was defined as the occurrence of no seizures since at least the previous visit; at 12 months, it was considered as no seizures during the preceding 6 months, and at 3 and 6 months was defined as lack of seizures since baseline or the 3-month visit, respectively. Safety and tolerability outcomes included the rate of treatment discontinuation due to AEs and the incidence of AEs considered BRV-related by participating physicians.
Exploratory analyses were performed to evaluate the impact of LEV status, i.e., prior LEV treatment (never LEV use, prior LEV use and discontinuation/switch to BRV due to insufficient efficacy, prior LEV use and discontinuation/ switch to BRV due to AEs), concomitant use of strong EiASMs, and concomitant use of SCBs.

\subsection{Statistical Analysis}

Values were presented as median [interquartile range] for continuous variables and number (\%) of subjects for categorical variables. Comparisons were made using the Mann-Whitney test, Dunn's test or Chi-square test, as appropriate. Simple and multivariable logistic regression models were performed to identify baseline characteristics of patients associated with 12-month seizure freedom. Preselected independent variables were age, sex, number of previous ASMs, number of concomitant ASMs, baseline monthly seizure frequency, and LEV status [16-18]; age, number of previous ASMs, number of concomitant ASMs, and baseline monthly seizure frequency were entered into regression models as continuous variables, and sex and LEV status as categorical variables.

Results were considered significant for $p$ values $<0.05$ (two-sided). Data analysis was performed using STATA/IC 13.1 (StataCorp LLC, College Station, TX, USA). The study is reported according to the Strengthening the Reporting of Observational Studies in Epidemiology (STROBE) guidelines [19].

\section{Results}

Of 1325 patients initially identified, 71 were excluded as diagnosed with generalized, combined or unknown epilepsy and 225 because follow-up after initiating BRV was $<1$ year at the data cut-off point for this report. Accordingly, 1029 patients with focal epilepsy were included. The median age of the patients was 45 years [33-56], and 487 $(47.3 \%)$ were males. Patients had a history of a median of 5 [3-8] lifetime ASMs, and BRV was added to a median of 2 [1-3] concomitant ASMs; the most common concomitant ASMs were carbamazepine, valproic acid and lacosamide. The median baseline seizure frequency was 5 [2-18], and 35 (3.4\%) patients were seizure-free during the 3 months before starting BRV. The baseline characteristics of participants are summarized in Table 1 and details about the concomitant ASMs are shown in Table 2.

The median daily dose of BRV at 3 months was $100 \mathrm{mg}$ [100-200], $150 \mathrm{mg}$ [100-200] at 6 months, and $150 \mathrm{mg}$ [100-200] at 12 months. At 12 months, 169 (16.4\%) patients were seizure-free and $383(37.2 \%)$ patients had their seizure 
frequency reduced by $50 \%$ or more in comparison with baseline. The rates of seizure response, seizure freedom and seizure worsening at 3 and 6 months are shown in Fig. 1.

The baseline characteristics of patients by LEV status are presented in Table 3. Patients with prior LEV use and withdrawal due to insufficient efficacy had a longer duration of epilepsy, were treated with more concomitant ASMs, and had higher seizure frequency at baseline than LEVnaive patients. At 12 months, the rate of seizure freedom was 59/265 (22.3\%) in LEV-naive patients, 34/481 (7.1\%) in patients with prior LEV use and discontinuation/switch to BRV due to insufficient efficacy, and $67 / 215$ (31.2\%) in patients with prior LEV use and discontinuation/switch to BRV due to AEs $(p<0.001)$; the corresponding values for

Table 1 Baseline characteristics of patients

\begin{tabular}{ll}
\hline Characteristics & Patients $[n=1029]$ \\
\hline Age, years & $45(33-56)$ \\
Male sex & $487(47.3)$ \\
Age at epilepsy onset, years $\left[N=1028^{\mathrm{a}}\right]$ & $13(6-26)$ \\
Duration of epilepsy, years $\left[N=1028^{\mathrm{a}}\right]$ & $25(13-38)$ \\
Type of seizure $\left[N=916^{\mathrm{a}}\right]$ & \\
Focal onset & $679(74.1)$ \\
Focal to bilateral tonic-clonic & $171(18.7)$ \\
Focal onset and focal to bilateral tonic-clonic ${ }^{\mathrm{b}}$ & $66(7.2)$ \\
Etiology & \\
Structural & $553(53.7)$ \\
Genetic & $40(3.9)$ \\
Immune & $11(1.1)$ \\
Infectious & $28(2.7)$ \\
Unknown & $397(38.6)$ \\
Number of previous ASMs $\left[N=1023^{\mathrm{a}}\right]$ & $5(3-8)$ \\
Levetiracetam status $\left[N=1022^{\mathrm{a}}\right]$ & \\
Never used & $265(25.9)$ \\
Prior use/prescribed at baseline & $757(74.1)$ \\
Number of concomitant ASMs $\left[N=1028^{\mathrm{a}}\right]$ & $2(1-3)$ \\
Concomitant use of EiASM(s) at baseline & $487(54.1)$ \\
{$\left[N=901^{\mathrm{a}}\right]$} & \\
Concomitant use of SCB(s) at baseline & $771(85.6)$ \\
$\left.\quad N=901^{\mathrm{a}}\right]$ & $5(2-18)$ \\
Baseline monthly seizure frequency & \\
\hline & \\
&
\end{tabular}

Data are expressed as median (IQR) for continuous variables, and $n$ (\%) for categorical variables

ASMs antiseizure medications, BRV brivaracetam, EiASMs enzymeinducing ASMs, IQR interquartile range, $S C B S$ sodium channel blockers

${ }^{\text {a }} N$ represents the total number of patients for whom data in question were available

${ }^{\mathrm{b}}$ Patients presenting both focal onset and focal to bilateral tonicclonic seizures

${ }^{\mathrm{c}}$ Based on the number of seizures during the 90 days before starting adjunctive BRV
Table 2 Concomitant antiseizure medications

\begin{tabular}{ll}
\hline Concomitant antiseizure medication & Patients $\left(N=901^{\mathrm{a}}\right)$ \\
\hline Carbamazepine & $359(39.8)$ \\
Valproic acid & $209(23.2)$ \\
Lacosamide & $176(19.5)$ \\
Phenobarbital & $172(19.1)$ \\
Lamotrigine & $170(18.9)$ \\
Perampanel & $112(12.4)$ \\
Benzodiazepines & $102(11.3)$ \\
Oxcarbazepine & $83(9.2)$ \\
Topiramate & $76(8.4)$ \\
Zonisamide & $53(5.9)$ \\
Eslicarbazepine acetate & $38(4.2)$ \\
Phenytoin & $32(3.6)$ \\
Primidone & $26(2.9)$ \\
Pregabalin & $18(2.0)$ \\
Rufinamide & $13(1.4)$
\end{tabular}

Data are expressed as $n(\%)$

${ }^{\mathrm{a}} N$ represents the total number of patients for whom data in question were available. Concomitant antiseizure medications taken by $>1 \%$ of patients are reported

$\geq 50 \%$ seizure frequency reduction were $47.9 \%, 29.7 \%$, and $42.8 \%$, respectively $(p<0.001)$.

At 12 months, there were no statistically significant differences in seizure freedom $(15.6 \%$ vs. $18.8 \% ; p=0.199)$ and seizure responder rates $(38.2 \%$ vs. $37.4 \% ; p=0.816)$ between patients receiving concomitant strong EiASMs versus patients treated with no strong EiASMs.

The baseline characteristics of patients by concomitant use of SCBs are shown in Table 4. Patients concomitantly taking SCBs had a longer duration of epilepsy, had been exposed to a higher number of prior ASMs, were treated with more concomitant ASMs, were more commonly treated with strong EiASMs, and had higher seizure frequency at baseline than patients concomitantly treated with no SCBs. There was a trend towards a higher 12-month seizure freedom rate in patients treated with add-on BRV and concomitant SCBs, but this was not statistically significant ( $p=0.341$ ), and there was a statistically significant $(p=0.006)$ higher number of responders to BRV in patients who were receiving SCBs than in those receiving no SCBs (Fig. 2).

Age [odds ratio (OR) 1.014, 95\% confidence interval (CI) $1.002-1.026 ; p=0.027$ ], number of previous ASMs (OR $0.75,95 \%$ CI $0.68-0.82 ; p<0.001)$, baseline monthly seizure count (OR 0.94, 0.91-0.97; $p<0.001$ ) and history of LEV withdrawal for tolerability issues (OR 2.25, 1.40-3.60; $p=0.001)$ were independent predictors of 12-month seizure freedom; patients with older age, lower number of lifetime ASMs, lower baseline seizure frequency and prior 
Fig. 1 Clinical response to adjunctive brivaracetam. Rates of seizure response, seizure freedom and seizure worsening at 3,6 and 12 months are reported. Seizure response was defined as a $\geq 50 \%$ reduction in seizure frequency in comparison with baseline seizure frequency, while seizure worsening was defined as an increase in seizure frequency of $>25 \%$ in comparison with baseline seizure frequency

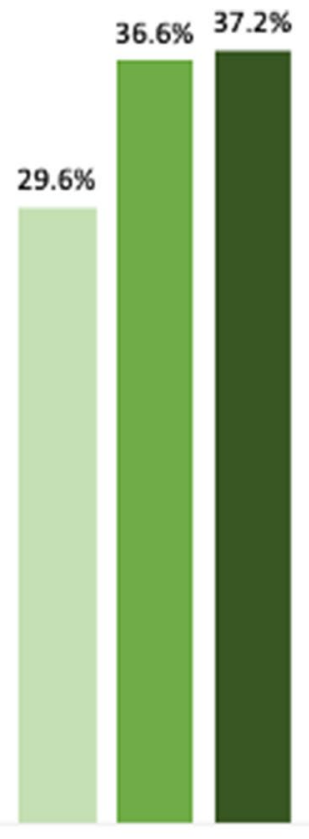

Seizure response

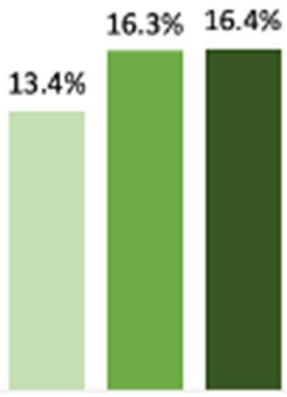

Seizure freedom

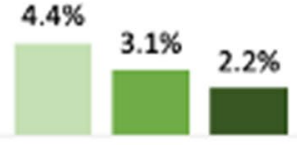

Seizure worsening

\section{3-month $\quad$ 6-month $\quad$ 12-month}

discontinuation of LEV due to adverse effects had a higher likelihood of being free from seizures (Table 5).

During the 1-year study period, 265 (25.8\%) patients of the cohort discontinued BRV. The reasons for BRV withdrawal were insufficient efficacy $(n=159,15.5 \%)$, AEs $(n=99,10.0 \%)$, and a combination of both $(n=5,0.5 \%)$; in one case, BRV was discontinued due to the patient's request and one patient died due to a cause unrelated to the treatment. Of 265 patients who withdrew BRV, 120 (45.3\%) had their treatment discontinued at 3 months, $71(26.8 \%)$ at 6 months, and 74 (27.9\%) at 12 months (Fig. 3).

The rate of treatment discontinuation for any cause was lower in patients who were LEV-naive $(22.3 \%)$ or had a history of LEV withdrawal due to adverse effects (18.6\%) than in those with prior LEV use and discontinuation/switch to BRV due to insufficient efficacy (30.6\%; $p=0.001$ ). Drug withdrawal due to poor efficacy occurred less commonly among patients who had never tried LEV $(13.2 \%)$ or had a history of LEV discontinuation due to adverse effects (8.8\%) than in patients who had used LEV and discontinued it due to insufficient efficacy $(20.0 \%$; $p<0.001)$. There were no statistically significant differences in the rates of BRV withdrawal for AEs according to LEV history ( $p=0.841$ ), and there were no statistically significant differences in the proportions of patients who withdrew BRV for any cause ( $p=0.763)$, insufficient efficacy $(p=0.090)$ or poor tolerability $(p=0.173)$ on the basis of concomitant use of strong EiASMs. Furthermore, there were no statistically significant differences in the rate of BRV withdrawal due to any cause $(p=0.188)$ or insufficient efficacy ( $p=0.899$ ) according to the concomitant use of SCBs; treatment discontinuation due to AEs was less common in patients treated with BRV and concomitant SCBs than in patients treated with BRV and no SCBs (8.8\% vs. $14.6 \% ; p=0.038)$.

AEs were reported by $30.1 \%$ of patients and were rated as mild $(75.4 \%)$, moderate $(24.2 \%)$ or severe $(0.4 \%)$ in intensity. The most common AEs observed in the study cohort included somnolence (6.6\%), nervousness and/or agitation (5.9\%), vertigo (3.6\%), fatigue (3.1\%), and headache (2.6\%) (Table 6). Among patients who switched from LEV to BRV due to AEs and for whom tolerability follow-up data were available, 3/26 (11.5\%) experienced new or persisting AEs.

There were no statistically significant differences in the occurrence of AEs according to LEV history $(p=0.833)$ and use of concomitant strong EiASMs $(p=0.071)$; AEs were less commonly observed in patients treated with BRV and concomitant SCBs than in patients treated with BRV and no SCBs $(28.9 \%$ vs. $39.8 \% ; p=0.017)$.

\section{Discussion}

The BRIVAFIRST provided interesting findings about the use of BRV in patients with focal epilepsy. The main results included (1) the large number of patients enrolled within the frame of an integrated national network; (2) the effectiveness of BRV in patients with focal seizures treated according 
Table 3 Baseline characteristics of patients according to levetiracetam status

\begin{tabular}{|c|c|c|c|}
\hline Characteristics & LEV-naïve $[n=265]$ & $\begin{array}{l}\text { LEV prior-insufficient } \\
\text { efficacy }[n=481]\end{array}$ & $\begin{array}{l}\text { LEV prior- } \\
\text { adverse events } \\
{[n=215]}\end{array}$ \\
\hline Age, years & $44(32-57)$ & $44(32-54)^{\ddagger}$ & $48(37-61)^{*}$ \\
\hline Male sex & $127(47.9)$ & $218(45.3)$ & $111(51.6)$ \\
\hline Age at epilepsy onset, years $\left[N=960^{\mathrm{a}}\right]$ & $14(5-29)$ & $12(5-20)^{*}$ & $18(7-44)^{*}$ \\
\hline Duration of epilepsy, years $\left[N=960^{\mathrm{a}}\right]$ & $23(10-36)$ & $26(16-39)^{* \ddagger}$ & $20(9-37)$ \\
\hline \multicolumn{4}{|l|}{ Type of seizure $\left(N=855^{\mathrm{a}}\right)$} \\
\hline Focal onset & $176(74.3)$ & $318(75.2)$ & $142(72.8)$ \\
\hline Focal to bilateral tonic-clonic & $52(21.9)$ & $71(16.8)$ & $34(17.4)$ \\
\hline Focal onset and focal to bilateral tonic-clonic ${ }^{\mathrm{b}}$ & $9(3.8)$ & $34(8.0)$ & $19(9.7)$ \\
\hline \multicolumn{4}{|l|}{ Etiology } \\
\hline Structural & $148(55.9)$ & $233(48.4)$ & $132(61.4)$ \\
\hline Genetic & $8(3.0)$ & $17(3.5)$ & $9(4.2)$ \\
\hline Immune & $2(0.8)$ & $5(1.0)$ & $4(1.9)$ \\
\hline Infectious & $7(2.6)$ & $15(3.1)$ & $2(0.9)$ \\
\hline Unknown & $100(37.7)$ & $211(43.9)$ & $68(31.6)$ \\
\hline Number of previous ASMs $\left[N=956^{\mathrm{a}}\right]$ & $3(2-5)$ & $7(5-9)^{* \dagger}$ & $5(3-7)^{*}$ \\
\hline Number of concomitant ASMs $\left[N=960^{\mathrm{a}}\right]$ & $2(1-2)$ & 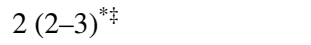 & $2(1-3)$ \\
\hline Concomitant use of $\operatorname{EiASM}(\mathrm{s})$ at baseline $\left[N=839^{\mathrm{a}}\right]$ & $113(48.7)$ & $237(57.3)$ & $107(55.4)$ \\
\hline Concomitant use of $\mathrm{SCB}(\mathrm{s})$ at baseline $\left[N=839^{\mathrm{a}}\right]$ & $193(83.2)$ & $359(86.7)$ & $172(89.1)$ \\
\hline Baseline monthly seizure frequency ${ }^{\mathrm{c}}$ & $5(2-15)$ & $7(3-20)^{* *}$ & $4(1-12)^{*}$ \\
\hline
\end{tabular}

Data are expressed as median (IQR) for continuous variables, and $n(\%)$ for categorical variables

$A S M s$ antiseizure medications, BRV brivaracetam, EiASMs enzyme-inducing ASMs, IQR interquartile range, LEV levetiracetam, SCBs sodium channel blockers

${ }^{*} p<0.05$ versus the LEV-naive group

${ }^{\ddagger} p<0.05$ versus the LEV prior-adverse events group

${ }^{a} N$ represents the total number of patients for whom data in question were available

${ }^{b}$ Patients presenting both focal onset and focal to bilateral tonic-clonic seizures

${ }^{\mathrm{c}}$ Based on the number of seizures during the 90 days before starting adjunctive BRV

to everyday clinical practice, including patients who have previously failed LEV; and (3) the higher responder rate observed among patients treated with BRV and concomitant SCBs than in those not receiving SCBs.

Among the studies aimed at evaluating the response to BRV add-on therapy in real-world clinical practice, the BRIVAFIRST is characterized by a larger population. This experience stemmed from the belief that a functional network engaged in building reliable evidence from real-world data may actually contribute to characterize the drug profile. Typically, regulatory trials deviate markedly from routine clinical practice, and the restrictive eligibility criteria, rigid titration and dosing schedules, and short follow-up duration limit the generalizability of the results [20]. In this regard, data generated in a real-life setting reflect physicians' experience and daily challenges. Studies performed in a naturalistic context do not only assess the efficacy and tolerability of ASMs under real-world conditions but also offer new insights on issues not addressed or left unanswered by pivotal trials.
In the cohort of patients included in the BRIVAFIRST who have a long disease duration, a considerable number of previously failed ASMs, and a high number of ongoing ASMs, the rates of seizure freedom and seizure response at 12-month follow-up were $16 \%$ and $37 \%$, respectively. These figures indicate the efficacy of BRV to control seizures when added to the pre-existing therapeutic regimen in everyday clinical practice in patients with difficult-to-treat epilepsy, and confirm previous evidence [21-28]. In line with other ASMs [29, 30], an inverse relationship between response to adjunctive BRV and the number of lifetime medications was observed, confirming that the higher the number of failed treatments, the lower the likelihood that the patient may benefit from subsequent interventions [31]. Likewise, the higher seizure frequency at baseline can act as a marker of the intrinsic severity of epilepsy.

Age was also an independent predictor of seizure freedom, with older age being associated with a greater probability of seizure freedom at 1 year after starting BRV. 
Table 4 Baseline characteristics of patients according to concomitant use of sodium channel blockers

\begin{tabular}{lll}
\hline Characteristics & $\begin{array}{l}\text { Concomitant use of SCBs } \\
{[n=771]}\end{array}$ & $\begin{array}{l}\text { Concomitant use of no SCBs } \\
{[n=130]}\end{array}$ \\
\hline Age, years & $45(34-56)$ & $46(30-63)$ \\
Male sex & $364(47.2)$ & $59(45.4)$ \\
Age at epilepsy onset, years & $13(6-26)$ & $15(7-40)$ \\
Duration of epilepsy, years & $25(13-38)$ & $20(9-33)$ \\
Type of seizure $\left[N=857^{\mathrm{a}}\right]$ & & $81(66.4)$ \\
Focal onset & $543(73.9)$ & $32(26.2)$ \\
Focal to bilateral tonic-clonic & $136(18.5)$ & $9(7.4)$ \\
Focal onset and focal to bilateral tonic-clonic ${ }^{\mathrm{b}}$ & $56(7.6)$ & $537(40.8)$ \\
Etiology & & $13(10.0)$ \\
Structural & $425(55.1)$ & $3(2.3)$ \\
Genetic & $20(2.6)$ & $3(2.3)$ \\
Immune & $7(0.9)$ & $58(44.6)$ \\
Infectious & $25(3.2)$ & $4(2-8)$ \\
Unknown & $294(38.1)$ & $1(1-2)$ \\
Number of previous ASMs $\left[N=898^{\mathrm{a}}\right]$ & $5(3-8)$ & $39(30.0)$ \\
Number of concomitant ASMs & $2(1-3)$ & $4(1-10)$ \\
Concomitant use of EiASM(s) at baseline & $448(58.1)$ & 0.006 \\
Baseline monthly seizure frequency & $6(2-20)$ & 0.135 \\
\hline
\end{tabular}

Data are expressed as median (IQR) for continuous variables, and $n(\%)$ for categorical variables

$A S M s$ antiseizure medication, $B R V$ brivaracetam, EiASMs enzyme-inducing ASMs, IQR interquartile range, $S C B s$ sodium channel blockers

${ }^{\text {a }} N$ represents the total number of patients for whom data in question were available

${ }^{b}$ Patients presenting both focal onset and focal to bilateral tonic-clonic seizures

${ }^{\mathrm{c}}$ Based on the number of seizures during the 90 days before starting adjunctive BRV

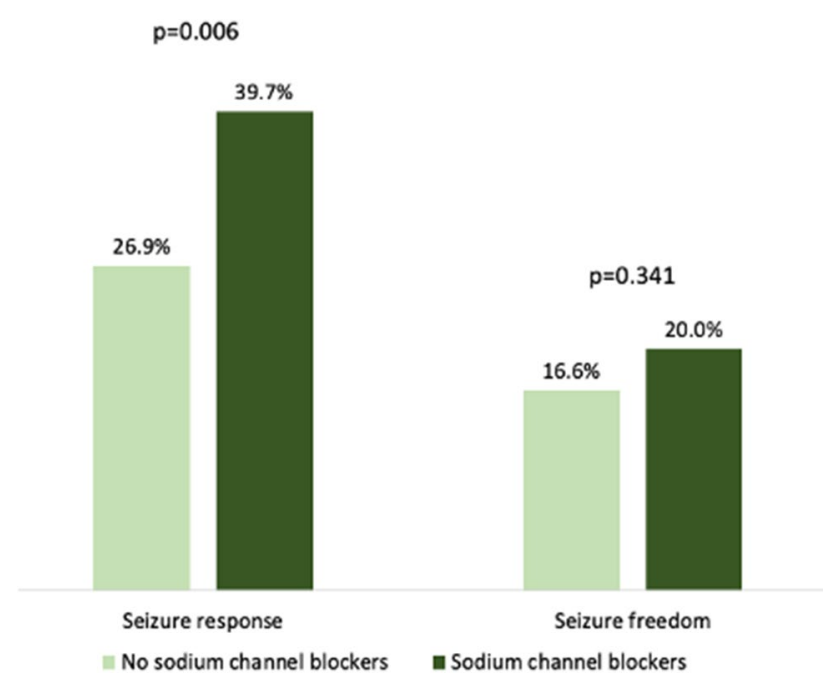

Fig. 2 Add-on brivaracetam efficacy according to concomitant use of sodium channel blockers. Rates of seizure response and seizure freedom at 12 months are reported according to concomitant use of sodium channel blockers (sodium channel blockers, $n=771$; no sodium channel blockers, $n=130$ ). Seizure response was defined as $a \geq 50 \%$ reduction in seizure frequency in comparison with baseline seizure frequency
Consistent with the greater proportion of responders already observed in patients aged $\geq 65$ years than in the younger subgroup [24], BRV might be considered as a viable choice for the treatment of focal seizures in the elderly. Differences in the epilepsy aetiology and metabolism across the classes of age need to be investigated to identify possible explanations, including drug blood levels that may be higher in elderly patients.

Given the structural similarities between BRV and LEV, the treatment efficacy according to prior LEV exposure is a matter of great interest and this study built up prior evidence through subgroup analyses by LEV historical use and reasons for LEV discontinuation. Adjunctive BRV was associated with seizure frequency reduction, both in patients with and patients without prior use of LEV. Of note, around $30 \%$ of patients with prior LEV use who discontinued it or switched to BRV due to insufficient efficacy had a reduction in baseline seizure frequency of $\geq 50 \%$, and $7 \%$ of patients were seizure-free at 12 months. The rates of seizure freedom and seizure response also highlighted the greater efficacy of BRV among LEV-naive patients than in patients who had previously discontinued LEV due to poor efficacy. It is worth noting that patients with prior LEV use and withdrawal due to insufficient efficacy had a longer duration of epilepsy, 
Table 5 Association between baseline characteristics and seizure freedom at 12 months

\begin{tabular}{|c|c|c|c|c|}
\hline \multirow[t]{2}{*}{ Dependent variable } & \multicolumn{2}{|l|}{ Unadjusted } & \multicolumn{2}{|l|}{ Adjusted $^{\mathrm{a}}$} \\
\hline & OR $(95 \% \mathrm{CI})$ & $p$ value & OR $(95 \% \mathrm{CI})$ & $p$ value \\
\hline Age & $1.03(1.02-1.04)$ & $<0.001$ & $1.014(1.002-1.026)$ & 0.027 \\
\hline Sex & $1.26(0.90-1.75)$ & 0.177 & $1.22(0.83-1.80)$ & 0.319 \\
\hline Number of previous ASMs & $0.67(0.62-0.72)$ & $<0.001$ & $0.75(0.68-0.82)$ & $<0.001$ \\
\hline Number of concomitant ASMs & $0.44(0.36-0.56)$ & $<0.001$ & $0.83(0.63-1.10)$ & 0.190 \\
\hline Baseline monthly seizure frequency & $0.90(0.87-0.93)$ & $<0.001$ & $0.94(0.91-0.97)$ & $<0.001$ \\
\hline \multicolumn{5}{|l|}{ Levetiracetam status ${ }^{\mathrm{b}}$} \\
\hline LEV prior-insufficient efficacy & $0.27(0.17-0.42)$ & $<0.001$ & $0.66(0.39-1.11)$ & 0.117 \\
\hline LEV prior-adverse events & $1.58(1.05-2.38)$ & 0.028 & $2.25(1.40-3.60)$ & 0.001 \\
\hline
\end{tabular}

Values are from logistic regression models

$A S M s$ antiseizure medications, $C I$ confidence interval, $L E V$ levetiracetam, $O R$ odds ratio

${ }^{a}$ Adjusted for age, sex, number of previous ASMs, number of concomitant ASMs, baseline monthly seizure frequency, and LEV status

${ }^{\mathrm{b}} \mathrm{LEV}$-naive group as the reference

Fig. 3 Patient disposition according to brivaracetam discontinuation

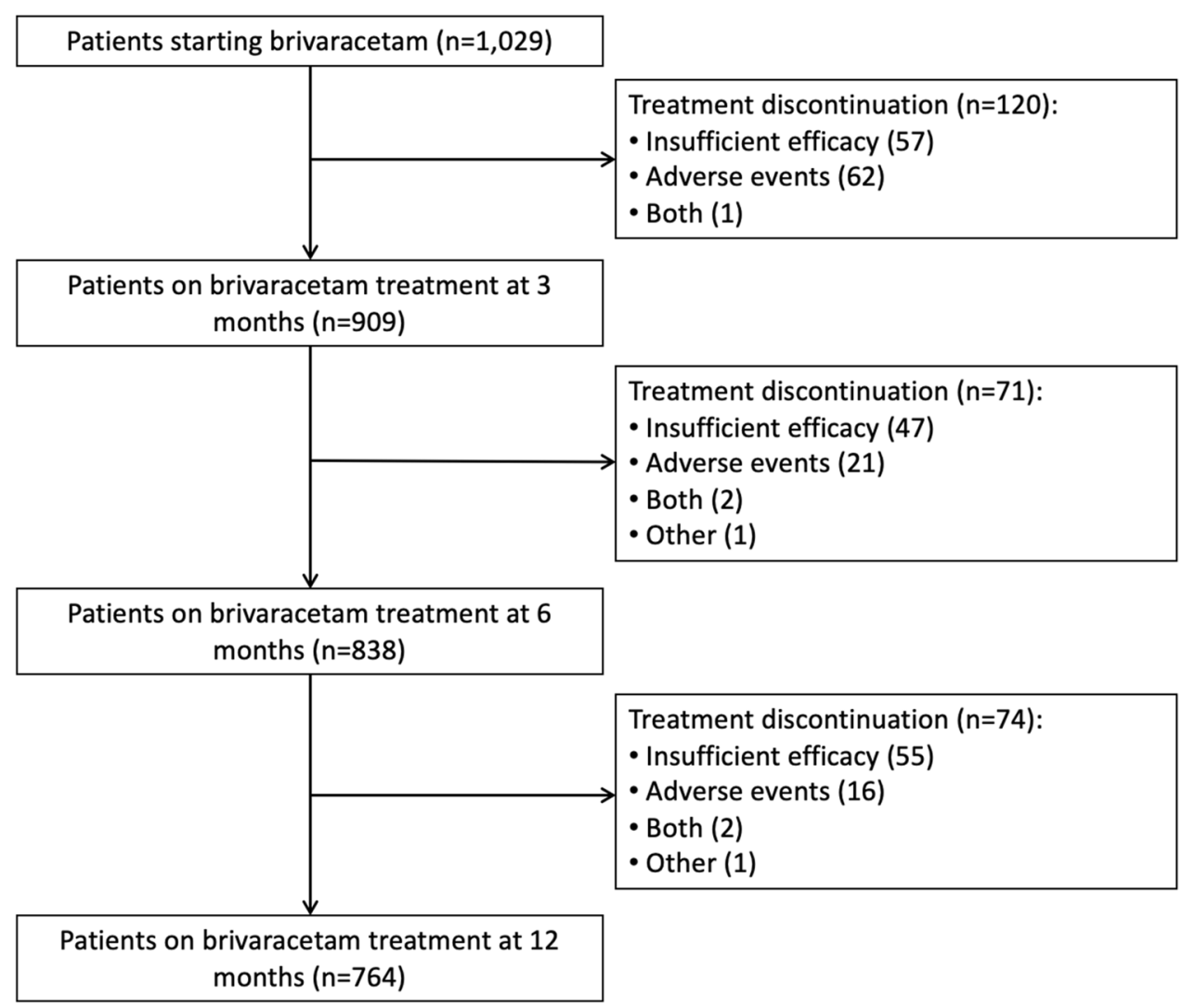

were treated with a higher number of concomitant ASMs, and had a higher seizure frequency at baseline than LEVnaive patients, suggesting that they could represent a group of more difficult-to-treat patients. However, the lack of information about serum concentrations of LEV could not allow evaluation of the actual status of treatment and whether there were cases of undertreatment. Similarly, in a pooled analysis of data from RCTs, BRV had greater efficacy in LEV-naïve patients than in patients with prior exposure to other commonly prescribed ASMs, including not only LEV but also carbamazepine, lamotrigine, and topiramate [32]. These findings strongly suggest that failure to respond to LEV does not preclude BRV treatment use. Despite similarities in their chemistry, differences exist between BRV and LEV. 
Table 6 Adverse events with brivaracetam treatment

\begin{tabular}{ll}
\hline Patients with adverse events & \\
$N^{\mathrm{a}}$ & 877 \\
$n(\%)$ & $264(30.1)$ \\
Most frequently reported adverse events (reported by $\geq 1 \%$ of \\
patients) \\
$N^{a}$ & 852 \\
Somnolence & $56(6.6)$ \\
Nervousness and/or agitation & $50(5.9)$ \\
Vertigo & $31(3.6)$ \\
Fatigue & $26(3.1)$ \\
Headache & $22(2.6)$ \\
Aggressiveness & $20(2.3)$ \\
Mood change & $20(2.3)$ \\
Dizziness & $19(2.2)$ \\
Sleep disturbances & $15(1.8)$ \\
Memory disturbance & $14(1.6)$ \\
\hline
\end{tabular}

Data are expressed as $n(\%)$

AEs reported by fewer than $1 \%$ of patients: nausea/vomiting, tremor (all $n=8,0.9 \%)$, stomach pain $(n=7,0.8 \%)$, disturbances in attention/concentration ( $n=6,0.7 \%)$, anxiety, diplopia/blurred vision (all $n=5,0.6 \%)$, weight increase $(n=4,0.5 \%)$, skin disorders, hair loss (all $n=3,0.4 \%$ ), fever, pharyngodynia, hyporexia (all $n=2,0.2 \%$ ), urinary disturbances, weight decrease, psychosis, tics, confusion, acouphene, constipation, abdominal pain (all $n=1,0.1 \%$ )

${ }^{\mathrm{a}} N$ represents the total number of patients for whom the data in question were available

Compared with LEV, BRV has higher affinity and selectivity for SV2A, and the differential interactions of the drugs with the target protein may provide the molecular correlate to their distinct pharmacodynamic properties and efficacy profiles [5]. Furthermore, the traditional modes of action of other ASMs do not contribute significantly to the antiseizure properties of BRV that, in contrast with LEV, have no effect on the $\alpha$-amino-3-hydroxy-5-methyl-4-isoxazolepropionic acid (AMPA) receptor and high-voltage-activated calcium channels at therapeutically relevant concentrations [33].

During the 1-year study period, the overall rate of treatment discontinuation was about $25 \%$, which was similar to that observed in other studies focusing on BRV and newer ASMs in clinical practice, including eslicarbazepine, perampanel, and lacosamide [21-29, 34-36]. The main reason for drug withdrawal was inadequate efficacy, and BRV was interrupted within the first 3 months of treatment in almost half of the cases. Around one-third of patients reported AEs throughout the 12-month follow-up, which were mostly mild in intensity. The most frequently observed AEs were somnolence, vertigo, fatigue and headache, which substantially overlap the profile of adverse effects of the majority of the ASMs [37]; nervousness and agitation were reported by $6 \%$ of patients and were the most common psychiatric AEs. These data confirmed the overall favourable tolerability profile of BRV when added to concomitant ASMs, and matched the already available evidence from both randomized and open-label studies [13, 21-28]. A slow-escalation dosing regimen may represent a useful strategy to avoid or minimize adverse effects as somnolence and fatigue.

Interesting and novel observations compared with prior studies emerged regarding the association between BRV and concomitant ASMs. Interestingly, the combination of BRV with SCBs, rather than no SCBs, resulted in better efficacy and tolerability and was associated with a higher rate of seizure responders and lower rates of AEs and treatment withdrawal due to AEs. To date, there are no experimental and clinical data about any advantageous combinations of BRV with other ASMs. As a general rule, favourable combinations usually consist of ASMs with different mechanisms of action [38], and additive or synergic efficacy without increased toxicity has been found when combining LEV with carbamazepine, oxcarbazepine and lacosamide [39]. Additional studies are warranted to specifically address this preliminary evidence regarding the favourable association of BRV with SCBs.

BRV has a low risk of clinically relevant DDIs. Strong EiASMs such as carbamazepine, phenytoin and phenobarbital have been shown to moderately reduce BRV plasma levels in the absence of a significant influence on clinical response and without the need for dose adjustment [7]. In the BRIVAFIRST, no statistically significant differences in the rates of seizure freedom, seizure responder, and AEs were found between patients receiving concomitant strong EiASMs and patients not treated with strong EiASMs. Although data on plasma drug concentrations were not available, these findings provided indications of a favourable pharmacokinetic profile of BRV, which can represent an advantageous characteristic in the case of poly drug regimens.

\subsection{Strengths and Limitations}

The main strengths of the BRIVAFIRST included the recruitment at multiple centres and the large sample size, which allowed exploratory subgroup analyses. Furthermore, the real-world setting, which reflects physicians' experience and judgements in adapting doses and discontinuing individual ASMs according to efficacy and tolerability concerns, provided a high degree of external validity and generalizability of the findings to everyday clinical practice. The study also presented some limitations, such as its open-label design and retrospective nature, which may have introduced potential sources of biases, the lack of details about specific etiologies and seizure frequency data by seizure subtypes, and the unavailability of serum levels of BRV and concomitant ASMs, which prevented exploring the individual interactions between drugs. The collection of AEs 
based on records of clinical visits rather than standardized questionnaires might have resulted in possible underreporting. Furthermore, the absence of a control group undergoing treatment with an alternative therapeutic option prevents drawing any conclusions about the comparative effectiveness of BRV and other ASMs.

\section{Conclusion}

The findings of the BRIVAFIRST provided real-world evidence on the effectiveness of BRV in patients with focal epilepsy irrespective of LEV history and concomitant ASMs, and suggested favourable therapeutic combinations. Further research is needed to explore the actual potential of BRV in specific aetiologies and epilepsy syndromes and also as early-add on treatment, and to provide more information for guiding clinical decisions.

Acknowledgements BRIVAFIRST Group Membership-Angela Alicino: Department of Basic Medical Sciences, Neurosciences and Sense Organs - University Hospital of Bari "A. Moro"; Michele Ascoli: Department of Medical and Surgical Sciences, Magna Græcia University of Catanzaro, Catanzaro, Italy; Giovanni Assenza: Dipartimento di Neurologia, Neurofisiopatologia, Neurobiologia, Università Campus Bio-Medico, Roma, Italy; Federica Avorio: Department of Human Neurosciences, Policlinico Umberto I, Sapienza University of Rome, Italy; Valeria Badioni: Neurology Unit, Maggiore Hospital, ASST Lodi, Lodi, Italy; Paola Banfi: Circolo Hospital, Fondazione Macchi - ASST Settelaghi, Varese, Italy; Emanuele Bartolini: USL Central Tuscany, Neurology Unit, Nuovo Ospedale Santo Stefano, Prato, Italy; Luca Manfredi Basili: Department of Human Neurosciences, Policlinico Umberto I, Sapienza University of Rome, Italy; Vincenzo Belcastro: Neurology Unit, Maggiore Hospital, ASST Lodi, Lodi, Italy; Simone Beretta: San Gerardo Hospital, ASST Monza, Italy; Irene Berto: Department of Human Neurosciences, Policlinico Umberto I, Sapienza University of Rome, Italy; Martina Biggi: Department Neurology 2, Careggi University Hospital, Florence, Italy; Giuseppe Billo: Epilepsy Center, UOC Neurology, AULSS 8 Vicenza, Vicenza, Italy; Giovanni Boero: Complex Structure of Neurology, SS Annunziata Hospital, Taranto, Italy; Paolo Bonanni: IRCCS Medea Scientific Institute, Epilepsy Unit, Conegliano, Treviso, Italy; Jole Bongorno: Neurology Unit, Giovanni Paolo II Hospital, Ragusa, Italy; Francesco Brigo: Department of Neurology, Hospital of Merano (SABES-ASDAA), Merano-Meran, Italy; Emanuele Caggia: Neurology Unit, Giovanni Paolo II Hospital, Ragusa, Italy; Claudia Cagnetti: Neurological Clinic, Department of Experimental and Clinical Medicine, Marche Polytechnic University, Ancona, Italy; Carmen Calvello: Dipartimento di Neurologia, Ospedale Santa Maria della Misericordia, Università di Perugia, Perugia, Italy; Emanuele Cerulli Irelli: Department of Human Neurosciences, Policlinico Umberto I, Sapienza University of Rome, Italy; Edward Cesnik: Neurology Unit, AOU Ferrara, Ferrara, Italy; Gigliola Chianale: Neurology Unit, San Giovanni Bosco Hospital, Turin, Italy; Domenico Ciampanelli: Institute of Clinical Neurophysiology, Department of Neuroscience, Policlinico Riuniti, Foggia, Italy; Roberta Ciuffini: Department of Life, Health, and Environmental Sciences, University of L'Aquila, Epilepsy Center, Ospedale San Salvatore, L'Aquila, Italy; Dario Cocito: Presidio Sanitario Major, Istituti Clinici Scientifici Maugeri, Turin, Italy; Donato Colella: Department of Human Neurosciences, Policlinico Umberto I, Sapienza University of Rome, Italy; Margerita Contento: Department Neurology 2, Careggi
University Hospital, Florence, Italy; Cinzia Costa: Dipartimento di Neurologia, Ospedale Santa Maria della Misericordia, Università di Perugia, Perugia, Italy; Eduardo Cumbo: Neurodegenerative Disorders Unit, Azienda Sanitaria Provinciale di Caltanissetta, Caltanissetta, Italy; Alfredo D'Aniello: IRCCS Neuromed, Pozzilli, Italy, Francesco Deleo: Epilepsy Unit, Fondazione IRCCS Istituto Neurologico "Carlo Besta”, Milan, Italy; Jacopo C DiFrancesco: San Gerardo Hospital, ASST Monza, Italy; Giancarlo Di Gennaro: IRCCS Neuromed, Pozzilli, Italy; Roberta Di Giacomo: Epilepsy Unit, Fondazione IRCCS Istituto Neurologico "Carlo Besta", Milan, Italy; Alessandra Di Liberto: Neurology Unit, San Giovanni Bosco Hospital, Turin, Italy; Elisabetta Domina: Neurology Unit, Maggiore Hospital, ASST Lodi, Lodi, Italy; Francesco Donato: Epilepsy Centre, Neurology Unit, Venice, Italy; Fedele Dono: Department of Neuroscience, Imaging and Clinican Science, "G. D'Annunzio" University" of Chieti-Pescara, Italy; Vania Durante: Neurology Unit, "Perrino" Hospital, Brindisi, Italy; Oasi Research Institute - IRCCS, Troina, Italy; Anna Estraneo: IRCCS Fondazione Don Carlo Gnocchi, Florence, Italy; Giacomo Evangelista: Department of Neuroscience, Imaging and Clinican Science, "G. D’Annunzio" University" of Chieti-Pescara, Italy; Maria Teresa Faedda: Department of Human Neurosciences, Policlinico Umberto I, Sapienza University of Rome, Italy; Ylenia Failli: Department Neurology 2, Careggi University Hospital, Florence, Italy; Elisa Fallica: Neurology Unit, AOU Ferrara, Ferrara, Italy; Jinane Fattouch: Department of Human Neurosciences, Policlinico Umberto I, Sapienza University of Rome, Italy; Alessandra Ferrari: Division of Clinical Neurophysiology and Epilepsy Center, IRCCS Ospedale Policlinico San Martino, Genova, Italy; Florinda Ferreri: Department of Neurosciences, University of Padua, Padua, Italy; Davide Fonti: University of Cagliari, Cagliari, Italy; Francesco Fortunato: Dipartimento di neurologia, Università "Magna Græcia", Catanzaro, Italy; Nicoletta Foschi: Neurological Clinic, Department of Experimental and Clinical Medicine, Marche Polytechnic University, Ancona, Italy; Teresa Francavilla: Department of Basic Medical Sciences, Neurosciences and Sense Organs- University Hospital of Bari “A. Moro"; Rosita Galli: Neurology Unit, Department of Cardio-neuro-vascular Sciences, San Donato Hospital, Arezzo, Italy; Stefano Gazzina: Clinical Neurophysiology Unit, Epilepsy Center, Spedali Civili, Brescia, Italy; Loretta Giuliano: Department of Medical and Surgical Sciences and Advanced Technologies "G.F. Ingrassia", Section of Neurosciences, University of Catania, Catania, Italy; Francesco HabetswallnerDepartment of Clinical Neurophysiology, Cardarelli Hospital, Naples, Italy; Francesca Izzi: Epilepsy Centre, Neurology Unit, Department of Systems Medicine, Policlinico Tor Vergata, University of Rome "Tor Vergata", Rome, Italy; Benedetta Kassabian: Department of Neurosciences, University of Padua, Padua, Italy; Angelo Labate: Dipartimento di neurologia, Università "Magna Græcia", Catanzaro, Italy; Concetta Luisi: Department of Neurosciences, University of Padua, Padua, Italy; Matteo Magliani: Department Neurology 2, Careggi University Hospital, Florence, Italy; Giulia Maira: Department of Medical and Surgical Sciences and Advanced Technologies "G.F. Ingrassia", Section of Neurosciences, University of Catania, Catania, Italy; Luisa Mari: Epilepsy Centre, Neurology Unit, Department of Systems Medicine, Policlinico Tor Vergata, University of Rome "Tor Vergata", Rome, Italy; Daniela Marino: Neurology Unit, Department of Cardio-neuro-vascular Sciences, San Donato Hospital, Arezzo, Italy; Addolorata Mascia: IRCCS Neuromed, Pozzilli, Italy; Alessandra Mazzeo: Institute of Clinical Neurophysiology, Department of Neuroscience, Policlinico Riuniti, Foggia, Italy; Stefano Meletti: Department of Biomedical, Metabolic and Neural Science, University of Modena and Reggio Emilia, Modena, Italy; Alessandra Morano: Department of Human Neurosciences, Policlinico Umberto I, Sapienza University of Rome, Italy; Annacarmen Nilo: Clinical Neurology Department of Neurosciences University Hospital S. Maria della Misericordia, Udine, Italy; Biagio Orlando: Department of Human Neurosciences, Policlinico Umberto I, Sapienza University of Rome, Italy; Francesco Paladin: Epilepsy Centre, Neurology Unit, 
Venice, Italy; Maria Grazia Pascarella: Neurology Unit, Maggiore Hospital, ASST Lodi, Lodi, Italy; Chiara Pastori: Epilepsy Unit, Fondazione IRCCS Istituto Neurologico "Carlo Besta", Milan, Italy; Giada Pauletto: Neurology Unit Department of Neurosciences University Hospital S. Maria della Misericordia, Udine, Italy; Alessia Peretti: Epilepsy Center, UOC Neurology, AULSS 8 Vicenza, Vicenza, Italy; Gabriella Perri: Neurology Unit, ASST Rhodense, Milan, Italy; Marianna Pezzella: Department of Clinical Neurophysiology, Cardarelli Hospital, Naples, Italy; Marta Piccioli: Dipartimento di neurologia, Ospedale San Filippo Neri, Rome, Italy; Pietro Pignatta: Neurology and epilepsy unit Humanitas Gradenigo Hospital, Turin, Italy; Nicola Pilolli: Complex Structure of Neurology, SS Annunziata Hospital, Taranto, Italy; Francesco Pisani: Neurology Unit, Department of Clinical and Experimental Medicine, University of Messina, Italy; Laura Rosa Pisani: Neurology Unit, Cutroni-Zodda Hospital, Barcellona, Messina, Italy; Fabio Placidi: Epilepsy Centre, Neurology Unit, Department of Systems Medicine, Policlinico Tor Vergata, University of Rome "Tor Vergata", Rome, Italy; Patrizia Pollicino: IRCCS Centro Neurolesi Bonino-Pulejo, Messina, Italy; Vittoria Porcella: AOU Sassari, Sassari, Italy; Silvia Pradella: USL Central Tuscany, Neurology Unit, Nuovo Ospedale Santo Stefano, Prato, Italy; Monica Puligheddu: University of Cagliari, Cagliari, Italy; Stefano Quadri: Neurology Unit, ASST Papa Giovanni XXIII, Bergamo, Italy; Rui Quintas: Epilepsy Unit, Fondazione IRCCS Istituto Neurologico "Carlo Besta", Milan, Italy; Rosaria Renna: Epilepsy Outpatient Clinic for Adults "A. Cardarelli" Hospital, Naples, Italy; Jessica Rossi: Department of Biomedical, Metabolic and Neural Science, University of Modena and Reggio Emilia, Modena, Italy; Adriana Rum: Dipartimento di neurologia e neurofisiopatologia, Aurelia Hospital, Rome, Italy; Enrico Michele Salamone: Department of Human Neurosciences, Policlinico Umberto I, Sapienza University of Rome, Italy, Ersilia Savastano: Department of Human Neurosciences, Policlinico Umberto I, Sapienza University of Rome, Italy; Maria Sessa: IRCCS Neuromed, Pozzilli, Italy; Department of Basic Medical Sciences, Neurosciences and Sense Organs University Hospital of Bari “A. Moro”; David Stokelj: Neurology Clinic, ASUGI, Trieste, Italy; Elena Tartara: Epilepsy Center, IRCCS Mondino Foundation, Pavia, Italy; Mario Tombini: Dipartimento di Neurologia, Neurofisiopatologia, Neurobiologia, Università Campus Bio-Medico, Rome, Italy; Gemma Tumminelli: Epilepsy Center, Child Neuropsychiatry Unit, AAST Santi Paolo Carlo, Milan, Italy; Maria Ventura: Neurology Unit, Giovanni Paolo II Hospital, Ragusa, Italy; Ilaria Viganò: Epilepsy Center, Child Neuropsychiatry Unit, AAST Santi Paolo Carlo, Milan, Italy; Emanuela Viglietta: Neurology and epilepsy unit Humanitas Gradenigo Hospital, Turin, Italy; Aglaia Vignoli: Department of Health Sciences, Università degli Studi, Milan, Italy; Flavio Villani: Division of Clinical Neurophysiology and Epilepsy Center, IRCCS Ospedale Policlinico San Martino, Genova, Italy; Elena Zambrelli: Epilepsy Center, Child Neuropsychiatry Unit, AAST Santi Paolo Carlo, Milan, Italy; Lelia Zummo: Neurology and Stroke Unit, P.O. ARNAS-Civico, Palermo, Italy.

\section{Declarations}

Funding Open access funding provided by Università Politecnica delle Marche within the CRUI-CARE Agreement.

Conflict of interest Simona Lattanzi has received speaker's or consultancy fees from Angelini, Eisai, GW Pharmaceuticals, and UCB Pharma, and has served on advisory boards for Angelini, Arvelle Therapeutics, Bial, and GW Pharmaceuticals. Laura Canafoglia has received consultancy fee from Eisai. Maria Paola Canevini has received speaker's or consultancy fees from Bial, Eisai, Italfarmaco, Sanofi, and UCB Pharma. Sara Casciato has participated in pharmaceutical industry-sponsored symposia for Eisai, UCB Pharma and Lusofarmaco. Valentina Chiesa has received speaker's or consultancy fees from Eisai and UCB Pharma. Anna Teresa Giallonardo has received consulting fees or speaker honoraria from Eisai. Angela La Neve has received speaker's or consultancy fees from Eisai, Mylan, Bial, Sanofi, and UCB Pharma. Patrizia Pulitano has received consulting fees or speaker honoraria from UCB Pharma and Eisai. Pier Paolo Quarato has participated in pharmaceutical industry-sponsored clinical trials and symposia for UCB Pharma. Federica Ranzato has received speaker's fees from Eisai, UCB, and Livanova. Eleonora Rosati has received fees for participation in advisory board or scientific consultation from Eisai, GW Pharmaceuticals, Bial, and UCB Pharma. Laura Tassi has received speaker's or consultancy fees from Arvelle Therapeutics, Eisai and UCB Pharma. Carlo Di Bonaventura has received consulting fees or speaker honoraria from UCB Pharma, Eisai, GW Pharmaceuticals, Bial, and Lusopharma., Filippo Dainese, Giovanni De Maria, Giuseppe Didato, Giovanni Falcicchio, Martina Fanella, Edoardo Ferlazzo, Giacomo Fisco, Massimo Gangitano, Filippo Sean Giorgi, Oriano Mecarelli, Elisa Montalenti and Federico Piazza have no conflicts of interest to declare.

Ethics Approval This study was approved by the Ethical Committee at all participating sites and was conducted in accordance with the Declaration of Helsinki.

Consent to Participate Informed consent was obtained from every patient and/or their parent or legal representative.

Consent for Publication Not applicable.

Availability of Data and Material Anonymized data will be shared at the request of any qualified investigator.

Code Availability Not applicable.

Author Contributions SL designed and conceptualized the study, coordinated and supervised the data collection, carried out the data analyses, and drafted the manuscript. VC, EF, ALN, and EM designed and conceptualized the study, and coordinated and supervised the data collection. LC, MPC, SC, FD, GDM, GD, GF, MF, GF, MG, ATG, FSG, OM, FP, PP, PPQ, FR, ER and LT were involved in the acquisition of data. CDB designed and conceptualized the study, coordinated and supervised the data collection, and drafted the manuscript. All authors critically revised the manuscript for important intellectual content. All authors approved the final manuscript for submission and agree to be accountable for all aspects of the work.

Open Access This article is licensed under a Creative Commons Attribution-NonCommercial 4.0 International License, which permits any non-commercial use, sharing, adaptation, distribution and reproduction in any medium or format, as long as you give appropriate credit to the original author(s) and the source, provide a link to the Creative Commons licence, and indicate if changes were made. The images or other third party material in this article are included in the article's Creative Commons licence, unless indicated otherwise in a credit line to the material. If material is not included in the article's Creative Commons licence and your intended use is not permitted by statutory regulation or exceeds the permitted use, you will need to obtain permission directly from the copyright holder. To view a copy of this licence, visit http://creativecommons.org/licenses/by-nc/4.0/. 


\section{References}

1. Chen Z, Brodie MJ, Liew D, Kwan P. Treatment outcomes in patients with newly diagnosed epilepsy treated with established and new antiepileptic drugs: a 30-year longitudinal cohort study. JAMA Neurol. 2018;75:279-86.

2. Lattanzi S, Zaccara G, Giovannelli F, et al. Antiepileptic monotherapy in newly diagnosed focal epilepsy. A network meta-analysis. Acta Neurol Scand. 2019;139:33-41.

3. Laxer KD, Trinka E, Hirsch LJ, et al. The consequences of refractory epilepsy and its treatment. Epilepsy Behav. 2014;37:59-70.

4. Rogawski MA. Brivaracetam: a rational drug discovery success story. Br J Pharmacol. 2008; 154:1555-7.

5. Klein P, Diaz A, Gasalla T, Whitesides J. A review of the pharmacology and clinical efficacy of brivaracetam. Clin Pharmacol. 2018;10:1-22.

6. Brivaracetam. Highlight of prescribing information. https://www. briviact.com/briviact-PI.pdf. Accessed Jul 2021.

7. Moseley BD, Chanteux H, Nicolas JM, Laloyaux C, Gidal B, Stockis A. A review of the drug-drug interactions of the antiepileptic drug brivaracetam. Epilepsy Res. 2020;163:106327.

8. Moseley BD, Otoul C, Staelens L, Stockis A. Pharmacokinetic interaction of brivaracetam on other antiepileptic drugs in adults with focal seizures: Pooled analysis of data from randomized clinical trials. Epilepsy Res. 2019;158:106218.

9. Brodie MJ, Fakhoury T, McDonough B, Colson A, Stockis A, Elmoufti S, Whitesides J. Brivaracetam-induced elevation of carbamazepine epoxide levels: A post-hoc analysis from the clinical development program. Epilepsy Res. 2018;145:55-62.

10. Schoemaker R, Wade JR, Stockis A. Brivaracetam population pharmacokinetics and exposure-response modeling in adult subjects with partial-onset seizures. J Clin Pharmacol. 2016;56:1591-602.

11. Moseley BD, Nicolas JM, Yeo KR, Chanteux H, Rosa M, Watanabe S, Kervyn S, Stockis A. The effect of brivaracetam on the pharmacokinetics of phenytoin: in vivo, in vitro, and modeling studies [abstract no. 2.207]. The American Epilepsy Society Annual Meeting; 2-6 December 2016: Houston, TX.

12. Nicolas JM, Chanteux H, Rosa M, Watanabe S, Stockis A. Effect of gemfibrozil on the metabolism of brivaracetam in vitro and in human subjects. Drug Metab Dispos. 2012;40:1466-72.

13. Lattanzi S, Cagnetti C, Foschi N, et al. Brivaracetam add-on for refractory focal epilepsy: a systematic review and meta-analysis. Neurology. 2016;86:1344-52.

14. Fisher RS, Cross JH, French JA, Higurashi N, Hirsch E, Jansen FE, et al. Operational classification of seizure types by the International League Against Epilepsy: position paper of the ILAE Commission for Classification and Terminology. Epilepsia. 2017;58:522-30.

15. Johannessen SI, Landmark CJ. Antiepileptic drug interactions - principles and clinical implications. Curr Neuropharmacol. 2010;8:254-67.

16. Lattanzi S, Cagnetti C, Foschi N, Provinciali L, Silvestrini M. Eslicarbazepine acetate as adjunctive treatment in partial-onset epilepsy. Acta Neurol Scand. 2018;137:29-32.

17. Lattanzi S, De Maria G, Rosati E, Didato G, Chiesa V, Ranzato F, et al. Brivaracetam as add-on treatment in focal epilepsy: a realworld time-based analysis. Epilepsia. 2021;62:e1-6.

18. Lattanzi S, Cagnetti C, Foschi N, Provinciali L, Silvestrini M. Lacosamide monotherapy for partial onset seizures. Seizure. 2015;27:71-4.

19. von Elm E, Altman DG, Egger M, et al. STROBE Initiative. The Strengthening the Reporting of Observational Studies in Epidemiology (STROBE) statement: guidelines for reporting observational studies. Ann Intern Med. 2007;147:573-7.
20. Walker MC, Sander JW. Difficulties in extrapolating from clinical trial data to clinical practice: the case of antiepileptic drugs. Neurology. 1997;49:333-7.

21. Adewusi J, Burness C, Ellawela S, Emsley H, Hughes R, Lawthom $\mathrm{C}$, et al. Brivaracetam efficacy and tolerability in clinical practice: a UK-based retrospective multicenter service evaluation. Epilepsy Behav. 2020;106:106967.

22. Steinhoff BJ, Christensen J, Doherty CP, Majoie M, De Backer M, Hellot S, et al. Effectiveness and tolerability of adjunctive brivaracetam in patients with focal seizures: second interim analysis of 6-month data from a prospective observational study in Europe. Epilepsy Res. 2020;165:106329.

23. Menzler K, Mross P, Rosenow F, Schubert-Bast S, Willems LM, Zahnert F, et al. First clinical postmarketing experiences in the treatment of epilepsies with brivaracetam: a retrospective observational multicentre study. BMJ Open. 2019;9:030746.

24. Villanueva V, López-González F, Mauri J, Rodriguez-Uranga J, Olivé-Gadea M, Montoya J, et al. BRIVA-LIFE study group: a multicenter retrospective study of the long-term use of brivaracetam in clinical practice. Acta Neurol Scand. 2018;139:360-8.

25. Hirsch M, Hintz M, Specht A, Schulze-Bonhage A. Tolerability, efficacy and retention rate of brivaracetam in patients previously treated with levetiracetam: a monocenter retrospective outcome analysis. Seizure. 2018;61:98-103.

26. Zahnert F, Krause K, Immisch I, Habermehl L, Gorny I, Chmielewska I, et al. Brivaracetam in the treatment of patients with epilepsy_first clinical experiences. Front Neurol. 2018;9:38.

27. Steinhoff BJ, Bacher M, Bucurenciu I, Hillenbrand B, Intravooth $\mathrm{T}$, Kornmeier R, et al. Real-life experience with brivaracetam in 101 patients with difficult-to-treat epilepsy: a monocenter survey. Seizure. 2017;48:11-4

28. Steinig I, von Podewils F, M.ddel G, Bauer S, Klein KM, Paule E, et al. Postmarketing experience with brivaracetam in the treatment of epilepsies: a multicenter cohort study from Germany. Epilepsia. 2017;58:1208-16

29. Villanueva V, Holtkamp M, Delanty N, et al. Euro-Esli: a European audit of real-world use of eslicarbazepine acetate as a treatment for partial-onset seizures. J Neurol. 2017;264:2232-48.

30. Runge U, Arnold S, Brandt C, et al. A noninterventional study valuating the effectiveness and safety of lacosamide added to monotherapy in patients with epilepsy with partial-onset seizures in daily clinical practice: the VITOBA study. Epilepsia. 2015;56:1921-30.

31. Schiller Y, Najjar Y. Quantifying the response to antiepileptic drugs. Effect of past treatment history. Neurology. 2008;70:54-65.

32. Asadi-Pooya AA, Sperling MR, Chung S, Klein P, Diaz A, Elmoufti $\mathrm{S}$, et al. Efficacy and tolerability of adjunctive brivaracetam in patients with prior antiepileptic drug exposure: a post-hoc study. Epilepsy Res. 2017;131:70-5.

33. Klitgaard H, Matagne A, Nicolas JM, Gillard M, Lamberty Y, De Ryck M, et al. Brivaracetam: rationale for discovery and preclinical profile of a selective SV2A ligand for epilepsy treatment. Epilepsia. 2016;57:538-48.

34. Lattanzi S, Cagnetti C, Foschi N, Ciuffini R, Osanni E, Chiesa $\mathrm{V}$, et al. Adjunctive perampanel in older patients with epilepsy: a multicenter study of clinical practice. Drugs Aging. 2021;38:603-10.

35. Wehner T, Mannan S, Turaga S, et al. Retention of perampanel in adults with pharmacoresistant epilepsy at a single tertiary care center. Epilepsy Behav. 2017;73:106-10.

36. Villanueva V, López-Gomáriz E, López-Trigo J, et al. Rational polytherapy with lacosamide in clinical practice: results of a Spanish cohort analysis RELACOVA. Epilepsy Behav. 2012;23:298-304. 
37. Zaccara G, Gangemi PF, Cincotta M. Central nervous system adverse effects of new antiepileptic drugs: a meta-analysis of placebo-controlled studies. Seizure. 2008;17:405-21.

38. Deckers CLP, Czuczwar SJ, Hekster YA, Keyser A, Kubova H, Meinardi $\mathrm{H}$, et al. Selection of antiepileptic drug polytherapy based on mechanisms of action: the evidence reviewed. Epilepsia. 2000;41:1364-74.
39. Verrotti A, Lattanzi S, Brigo F, et al. Pharmacodynamic interactions of antiepileptic drugs: From bench to clinical practice. Epilepsy Behav. 2020;104(Pt A):106939.

\section{Authors and Affiliations}

\section{Simona Lattanzi ${ }^{1}$ (D) Laura Canafoglia ${ }^{2} \cdot$ Maria Paola Canevini ${ }^{3,4} \cdot$ Sara Casciato ${ }^{5}$. Valentina Chiesa ${ }^{3}$. Filippo Dainese $^{6} \cdot$ Giovanni De Maria $^{7}$. Giuseppe Didato ${ }^{8}$. Giovanni Falcicchio ${ }^{9} \cdot$ Martina Fanella $^{10}$. Edoardo Ferlazzo ${ }^{11}$. Giacomo Fisco ${ }^{10}$. Massimo Gangitano ${ }^{12}$. Anna Teresa Giallonardo ${ }^{10}$. Filippo Sean Giorgi ${ }^{13,14}$. Angela La Neve ${ }^{9}$. Oriano Mecarelli ${ }^{10}$. Elisa Montalenti ${ }^{15} \cdot$ Federico Piazza $^{16} \cdot$ Patrizia Pulitano $^{10}$. Pier Paolo Quarato ${ }^{5}$. Federica Ranzato ${ }^{17}$. Eleonora Rosati ${ }^{18} \cdot$ Laura Tassi $^{19} \cdot$ Carlo Di Bonaventura $^{10}$ on behalf of BRIVAFIRST Group Membership}

1 Neurological Clinic, Department of Experimental and Clinical Medicine, Marche Polytechnic University, Via Conca 71, 60020 Ancona, Italy

2 Department of Epileptology, Fondazione IRCCS Istituto Neurologico Carlo Besta, Milan, Italy

3 Epilepsy Center, Child Neuropsychiatry Unit, AAST Santi Paolo Carlo, Milan, Italy

4 Department of Health Sciences, Università degli Studi, Milan, Italy

5 IRCCS Neuromed, Pozzilli, Italy

6 Epilepsy Centre, Neurology Unit, Venice, Italy

7 Clinical Neurophysiology Unit, Epilepsy Center, Spedali Civili, Brescia, Italy

8 Epilepsy Unit, Fondazione IRCCS Istituto Neurologico "Carlo Besta", Milan, Italy

9 Department of Basic Medical Sciences, Neurosciences and Sense Organs, University Hospital of Bari "A. Moro", Bari, Italy

10 Department of Human Neurosciences, Policlinico Umberto I, Sapienza University of Rome, Rome, Italy
11 Department of Medical and Surgical Sciences, Magna Græcia University of Catanzaro, Catanzaro, Italy

12 Department of Biomedicine, Neuroscience, and advanced Diagnostic (BIND), University of Palermo, Palermo, Italy

13 Department of Translational Research on New Technologies in Medicine and Surgery, University of Pisa, Pisa, Italy

14 Neurology Unit, Pisa University Hospital, Pisa, Italy

15 Epilepsy Center, AOU Città della Salute e della Scienza di Torino, Turin, Italy

16 Department of Neurosciences, Rita Levi Montalcini, University of Turin, Turin, Italy

17 Epilepsy Center, UOC Neurology, AULSS, 8 Vicenza, Vicenza, Italy

18 Department Neurology 2, Careggi University Hospital, Florence, Italy

19 C. Munari” Epilepsy Surgery Centre, Niguarda Hospital, Milan, Italy 